\title{
STRATEGIC CHOICE HANDICAPS WHEN FEMALES SEEK HIGH MALE NET VIABILITY*
}

\author{
Georg Nöldeke \\ Department of Economics \\ University of Bonn \\ Adenauerallee 24-26 \\ 53113 Bonn, Germany
}

\author{
Larry Samuelson \\ Department of Economics \\ University of Wisconsin \\ 1180 Observatory Drive \\ Madison, Wisconsin 53706-1320 USA
}

July 17, 2001

Running Headline: Strategic Choice Handicaps

Contact author: Larry Samuelson, Department of Economics, University of Wisconsin, 1180

Observatory Drive, Madison, WI, 53717. Email: LarrySam@ssc.wisc.edu. Phone: 608-263-7791 (voice), 608-262-2033 (fax).

\section{Summary}

We examine a strategic-choice handicap model in which males send costly signals to advertise their quality to females. Females are concerned with the net viability of the male with whom they mate, where net viability is a function of the male's quality and signal. We identify circumstances in which a signaling equilibrium would require high-quality males to send signals so much larger than those of lower-quality males (to deter mimicry by the latter) as to yield lower net viabilities for the former. This causes females to shun males who send large signals, ensuring that there is no signaling equilibrium.

*We thank Carl Bergstrom, Ilan Eshel, Charles Godfray, Rufus Johnstone, Steve Stearns, and a referee for helpful comments. We thank the Deutsche Forschungsgemeinschaft, Graduiertenkolleg 629 at the University of Bonn, and the National Science Foundation for financial support. 


\title{
Strategic Choice Handicaps when Females Seek High Male Net Viability
}

\author{
by Georg Nöldeke and Larry Samuelson
}

\section{Introduction}

Zahavi's $(1975,1977)$ handicap principle was initially regarded skeptically. For instance, Maynard Smith (1976) observed that if males use a costly handicap to advertise their genetic quality, then females choosing high-quality males will bear offspring who inherit not only the advantageous genetic quality but also the fitness-reducing handicap. Maynard Smith argued that the deleterious effects of the handicap were likely to overwhelm the benefit of good genes, rendering the signal counterproductive as a means of revealing quality. This issue has been pursued by (among others) Eshel and Feldman (1991), Iwasa and Pomiankowski (1995), Iwasa, Pomiankowski and Nee (1991), Kirkpatrick (1986), Otto (1991), and Pomiankowski (1987a, 1987b) (see Kirkpatrick (1987) for a survey of earlier work). As noted by Pomiankowski (1987b, p. 124), this analysis is complicated by the conceptual and practical difficulties in separating the handicap principle from Fisher's sexual selection process.

Grafen (1990a, 1990b) departs from this line of work by examining a "strategic choice" handicap, in which high quality males confer a nonheritable advantage on females, such as increased fecundity or parental care. His motivation in constructing such a model was to separate the handicap principle from forces arising out of the Fisher process (Grafen, 1990b, pp. 473-474, 484-487). Grafen showed that equilibria exist in which males choose signals (i.e., handicaps) which reveal their quality. The evolutionary stability of such a signaling equilibrium hinges on the fact that signals are costly, so that low-quality males find it prohibitively expensive to mimic high-quality males. Rather than posing a fatal obstacle, the cost of a strategic handicap is the key to its success as a means of communication. Subsequent work has shown that strategic handicaps can form the basis for costly communication in a variety of contexts (see Godfray and 
Johnstone (2000) and Johnstone (1998) for surveys and see Eshel, Volovik and Sansone (2000) for an exploration of the interplay between the Fisher process and strategic handicaps). These include models of the advertising of quality (Grafen (1990a, 1990b), Johnstone (1995)) as well as models of the signaling of need (Godfray (1991, 1995a, 1995b), Johnstone and Grafen (1992), Kilner and Johnstone (1997), Maynard Smith (1991, 1994), Nöldeke and Samuelson (1999)).

With the exception of Eshel, Volovik and Sansone, these investigations have followed the bulk of Grafen's (1990a, 1990b) analysis in assuming that higher signals impose higher fitness costs on the (higher quality) males who send them, but impose no costs on the females who mate with these males. Hence, the adverse-advertising effect that Maynard Smith identified as a threat to the handicap principle has disappeared along with the Fisher effect. In this paper, we fill a gap in Grafen's analysis by examining an explicitly game-theoretic strategic handicap model in which females are concerned with the net viability of the male with whom they mate, where net viability is a function of the male's quality and the male's signal (Grafen, 1990a, pp. 525526). Maynard Smith's adverse-advertising effect thus reappears, though in a different guise. We derive a condition on the distribution of male qualities that is necessary and sufficient for the existence of a signaling equilibrium. When this condition is not satisfied, signaling requires that higher-quality males send much larger signals than males of lower quality, to deter mimicry by low-quality males. The signals required of high-quality males are so large as to reduce their net viability below that of lower-quality males, causing females to shun high signals and hence ensuring that males prefer not to signal. There is then no signaling equilibrium.

\section{The Model}

Zahavi's handicap principle potentially explains costly signaling in a variety of contexts. For concreteness, we follow Grafen (1990a, 1990b) in presenting a model in which males signal their quality to females. Section 4 discusses one of the many possible alternative applications.

We assume that each male $i$ is endowed at birth with a quality or condition $\theta_{i}$ that is independently drawn from the differentiable distribution $F$ with support $[0, \bar{\theta}]$ and density $f$. 
This condition is not observed by other males or females. Males survive to adulthood with certainty, at which point each male competes with $n-1$ other males for the attention of a female. The female observes a signal $s_{i}$ (which may depend on $\theta_{i}$ ) for each male $i$, and then selects one of the males with whom to mate.

A male who is not chosen by the female has an expected reproductive success or fitness of zero. If the female chooses a male whose condition is $\theta_{i}$ and signal is $s_{i}$, then the fitness of the chosen male is $\theta_{i}-s_{i}$ and the female's fitness is given by a strictly increasing function of $\theta_{i}-s_{i}$. Hence, females prefer males with high net viability $\theta_{i}-s_{i}$. Grafen $(1990 \mathrm{~b}, \mathrm{p} .476)$ suggests fecundity as a nongenetic benefit that males may confer on females, with our analysis then pertaining to a case in which fecundity is linked to the male's net viability rather than condition. Alternatively, the female may value the male's ability to provide parental care, which may be linked to net viability.

A strategy for a male is a function $s_{i}:[0, \bar{\theta}] \rightarrow \mathbb{R}_{+}$, with $s_{i}\left(\theta_{i}\right)$ identifying the signal the male would send if in condition $\theta_{i}$. A collection of such strategies is an equilibrium if each male's strategy $s_{i}(\cdot)$ maximizes the male's expected fitness, given the strategies of the female and other males, and if the female chooses, from any set of male signals, the signal that maximizes her expected fitness. We restrict our attention to signaling equilibria, in which each signal is sent by only one condition of male, so that signals reveal their senders' conditions. (Other equilibria are discussed at the end of the next section.) We concentrate on equilibria that are strict (i.e., equilibria in which there is a unique optimal signal for each male condition and amost surely a unique optimal female choice given the signals she observes) and hence evolutionarily stable (Selten, 1980).

A key observation is that in a signaling equilibrium, males sending larger signals must be more likely to be selected by the female, since otherwise they would not incur the extra cost of the higher signal. In addition, females "get what they want" in a strict signaling equilibrium: they can identify the characteristics of the males whose signals they observe and choose their (uniquely) preferred male, in this case the male with the highest net viability. Males sending 
higher signals must then have higher net viabilities, and hence must be males in higher condition, and females must choose the male sending the highest signal.

This link between high male condition and high signals characterizes any strict signaling equilibrium. Attention then turns to the question of when such equilibria exist. The essential question is whether males in higher condition send signals high enough to signal their quality without reducing their net viability. If female fitness depends only on male condition, this question is moot. If female fitness depends on net male viability, an affirmative answer is necessary (and sufficient) for a signaling equilibrium.

\section{Existence of Equilibrium}

Given that females choose the highest-signaling male, our model is much like an auction, in which the males bid (signal) to be chosen by the female. It follows from standard results in auction theory that the game has a unique candidate for a strict signaling equilibrium (Fudenberg and Tirole 1991, pp. 223-225, Maskin and Riley, 1986). All males adopt identical, differentiable strategies, with males in higher condition sending larger signals.

To derive the males' behavior in this candidate equilibrium, we begin with the observation that signals must be increasing in condition. If $s(\theta)$ is the (common) male strategy and a male in condition $\theta$ sends the signal $s(\hat{\theta})$ for some $\hat{\theta}$, then the male's fitness is

$$
(\theta-s(\hat{\theta})) F(\hat{\theta})^{n-1}
$$

where $(\theta-s(\hat{\theta}))$ is the net viability conditional on being chosen by the female and $F(\hat{\theta})^{n-1}$ is the probability that all other males are in condition less than $\hat{\theta}$ and hence send a signal less than $s(\hat{\theta})$, causing the female to select the male in question.

To calculate the optimal signal of a male in condition $\theta$ (given the strategy $s(\cdot)$ ), we first differentiate (1) with respect to $\hat{\theta}$ to obtain

$$
(\theta-s(\hat{\theta}))(n-1) F(\hat{\theta})^{n-2} f(\hat{\theta})-s^{\prime}(\hat{\theta}) F(\hat{\theta})^{n-1} .
$$


If $s(\theta)$ is to be an equilibrium, it must optimal for a male in condition $\theta$ to send signal $s(\theta)$, so that (2) must equal zero when $\hat{\theta}=\theta$. Rearranging the resulting equality gives

$$
s(\theta)(n-1) F(\theta)^{n-2} f(\theta)+s^{\prime}(\theta) F(\theta)^{n-1}=\theta(n-1) F(\theta)^{n-2} f(\theta) .
$$

Integrating both sides, we have

$$
s(\theta) F(\theta)^{n-1}=\int_{0}^{\theta}(n-1) \tilde{\theta} F(\tilde{\theta})^{n-2} f(\tilde{\theta}) d \tilde{\theta}
$$

or, equivalently

$$
s(\theta)=\frac{\int_{0}^{\theta}(n-1) \tilde{\theta} F(\tilde{\theta})^{n-2} f(\tilde{\theta}) d \tilde{\theta}}{F(\theta)^{n-1}},
$$

where (4) makes use of the fact that the male in condition 0 has a zero probability of being the highest signaler, and hence optimally sends a zero signal (Grafen, 1990a, p. 543). In any signaling equilibrium, the signals must be given by (5). Integrating the numerator by parts allows one to verify that equilibrium signals are increasing in male condition.

Signaling equilibria typically hinge upon the fact that males in higher condition find it relatively less costly to send higher signals, perhaps because increased signals impose a lower incremental mortality risk on such males. This relationship appears in our model in a different guise: males in higher condition derive greater benefits from higher signals. In particular, a higher signal reduces fitness conditional on being chosen by the female (given by $\theta_{i}-s_{i}$ ), but increases the chance of being chosen. A male in higher condition has more to gain from mating with the female and hence finds it more advantageous to increase the chance of mating by choosing a larger signal. Technically, this shows up in the fact that the derivative in (2) is larger for larger values of the condition $\theta_{i}$. Intuitively, we need only note that an increase in signal from 0.1 to 0.2 may be beneficial for a male in condition 1.0, but is surely disastrous for a male in condition 0.15 .

As we have explained at the end of the preceding section, a signaling equilibrium exists only if males sending higher signals have higher net viability, so that it is an equilibrium for the female to select the male sending the highest signal. Equilibrium net viability is given by

$$
\theta-s(\theta)=\theta-\frac{\int_{0}^{\theta}(n-1) \tilde{\theta} F(\tilde{\theta})^{n-2} f(\tilde{\theta}) d \tilde{\theta}}{F(\theta)^{n-1}}
$$




$$
=\frac{\int_{0}^{\theta} F(\tilde{\theta})^{n-1} d \tilde{\theta}}{F(\theta)^{n-1}},
$$

where (7) is obtained by performing integration by parts on the integral on the right side of (6). We thus have:

Proposition A strict signaling equilibrium exists if and only if the expression in (7) is strictly increasing in $\theta$, or equivalently, if and only if $\ln \int_{0}^{\theta} F(\tilde{\theta})^{n-1} d \tilde{\theta}$ is strictly concave.

A function $g(\theta)$ is said to be strictly $\log$ concave if and only if $\ln g(\theta)$ is strictly concave (An (1998), Bagnoli and Bergstrom (1989)). The existence of a strict signaling equilibrium thus hinges upon whether the distribution of male conditions is such that $\int_{0}^{\theta} F(\tilde{\theta})^{n-1} d \tilde{\theta}$ is strictly $\log$ concave.

A sufficient (but not necessary) condition for $\int_{0}^{\theta} F(\tilde{\theta})^{n-1} d \tilde{\theta}$ to be $\log$ concave is that the distribution of male conditions have a log concave density, since the integral of the distribution function raised to the power $n-1$ (i.e., $\int_{0}^{\theta} F(\tilde{\theta})^{n-1} d \tilde{\theta}$ ) will then be log concave (because $\log$ concavity is preserved both by being raised to any power $n-1$ greater than one and by integration (An, 1998, Lemma 3)). Many common densities are log concave (An, 1998, Bagnoli and Bergstrom, 1989). An exponential density marks the borderline between log concavity and log convexity, with log concave densities declining faster than does the exponential.

If the distribution of male conditions has a log concave density, then a signaling equilibrium will exist for any number $n$ of males involved in the signaling competition. The derivative of (7) with respect to $n$ is negative. Hence, as the number of males competing for the female increases, the equilibrium net viability arising out of the competition for a single female decreases for every condition of male.

The $\log$ concavity of $\int_{0}^{\theta} F(\tilde{\theta})^{n-1} d \tilde{\theta}$ can fail if the density concentrates its mass on males close to the lowest and highest condition. For example, Fig. 1 shows the density for the distribution function:

$$
F(\theta)=\left\{\begin{array}{lll}
\frac{1}{2}-\frac{1}{2}(1-\theta)^{\alpha+1} & \text { if } & \theta \in[0,1] \\
\frac{1}{2}+\frac{1}{2}(\theta-1)^{\alpha+1} & \text { if } & \theta \in[1,2]
\end{array} .\right.
$$


When there are two males involved in the competition for the female $(n=2)$ and $F(\theta)$ is given by (8), the $\log$ concavity of $\int_{0}^{\theta} F(\tilde{\theta})^{n-1} d \tilde{\theta}$ fails, and hence a signaling equilibrium fails to exist, for any $\alpha>1$. Males in relatively high condition face stiff competition from other males in similar condition, enhancing the payoff to aggressive signaling. The top panel in Fig. 2 accordingly shows that the signal $s(\theta)$ increases sharply in the male's condition $\theta$ for large values of the latter. In contrast, males in medium condition are unlikely to face competition from others in similar condition, making it less likely that higher signals will be effective and leading to relatively modest signaling. If signals are to honestly reveal condition, males in high condition must choose signals yielding a lower net viability than males in medium condition, as shown in the bottom panel of Fig. 2. But this ensures that there is no signaling equilibrium.

Although signaling equilibria are our primary concern, it is useful to note that there always exists an equilibrium in which no signals are sent and females ignore any signals that they happen to observe. There may also exist other equilibria, in which signals are partially informative or in which males' signals reveal their conditions but males in different condition send different signals that lead to identical equilibrium net viabilities, with the female choosing randomly among these net viabilities in the precise proportions required to support the equilibrium. Such equilibria are neither strict nor evolutionarily stable, and can be disrupted by drift in the female's strategy.

\section{Discussion}

Examining a strategic-choice handicap model in which females are concerned with net male viability, we have derived a necessary and sufficient condition for the existence of a signaling equilibrium. This condition, concerning the distribution of male qualities and ensuring that females prefer high-signaling males, is likely to be satisfied when the accompanying density is unimodal and likely to fail when it departs significantly from unimodality, such as in our example of a sharply U-shaped distribution.

If a signaling equilibrium exists in our model, whether females are concerned with male condition or with net male viability, its properties match those identified by Grafen (1990a)— 
males in higher condition send higher signals and (in the latter but not necessarily former case) have higher net viability. If females in our model do not bear the cost of male advertising, then a signaling equilibrium exists. Our contribution is the demonstration that when females are concerned with net viability, the existence of a signaling equilibrium in a model of strategic handicaps cannot be taken for granted. Grafen (1990a) circumvents this issue by working with a "model of the [strategic] model" that implicitly assumes the existence of an equilibrium in the underlying strategic model (Grafen, 1990b, pp. 515-516).

We have attempted to isolate the issues surrounding signaling and net viability by examining a very simple model. We could generalize the model so that a male survives to adulthood with a nonunitary probability, depending on his condition $\theta_{i}$, simply by respecifying $F\left(\theta_{i}\right)$ to be the distribution of surviving males. We could also allow net viability to be given by a general function $V\left(\theta_{i}, s_{i}\right)$ instead of $\theta_{i}-s_{i}$. The net viability $V\left(\theta_{i}, s_{i}\right)$ might then initially increase and subsequently decrease in the level of the signal, allowing us to capture situations in which signals are costly exaggerations of traits that are fitness-enhancing at low levels. In this case, the derivation of the equilibrium signal $s_{i}\left(\theta_{i}\right)$ would proceed by the same method as before, with the equilibrium condition that $\theta_{i}-s_{i}\left(\theta_{i}\right)$ be increasing (cf. (6)-(7)) then being replaced with the condition that $V\left(\theta_{i}, s_{i}\left(\theta_{i}\right)\right)$ is increasing in $\theta_{i}$. Next, nothing in our analysis must change if males can engage in multiple competitions for females, provided that the outcome of the current competition has no effect on subsequent opportunities. Finally, it would be interesting to allow the male's survival probability to depend upon both his condition $\theta_{i}$ and signal $s_{i}$. In this case, the male's fitness given by equation (1) would be multiplied by a survival probability (depending upon the male's condition and signal), and the distribution $F\left(\theta_{i}\right)$ would be replaced by an endogenously-determined distribution of surviving males' conditions that depends upon the distribution of males' conditions at birth and the equilibrium signals.

Our analysis suggests that seemingly excessive signaling costs should be observed either in cases where females care only about male condition, perhaps because males contribute only sperm to the reproductive venture (e.g. peacocks and birds of paradise), or in cases in which 
some mechanism other than the handicap principal (e.g., the Fisher process) lies behind the signals. Our model, with its limitations on equilibrium signal costs, is more likely to apply to those species in which males both signal their condition and make a contribution such as fecundity or parental care to the reproductive venture.

We have followed Grafen's lead in using males competing for females as the context in which to interpret our analysis. Like the handicap principle, however, our result is relevant for much more than sexual selection. For example, Zahavi has suggested that prey might signal their condition to potential predators (see, e.g., Dawkins (1989, p. 171)). Predator-prey interactions provide a natural setting in which the receiver's fitness depends upon both the condition and the signal of the sender. Predators seek prey in low condition (i.e., who will be easy to catch), while high-condition prey distinguish themselves by sending signals that have the cost of making escape more difficult. Depending upon the distribution of prey conditions, the signals required to support an equilibrium may be so costly as to make high-condition prey more inviting targets (the equivalent of reducing their net viability). This in turn will preclude an equilibrium in which prey signal their conditions to potential predators. Notice that situations such as predator-prey interactions, in which the signaler and receiver are of different species, are free of the genetic correlations potentially introduced by same-sex interactions (e.g. Pen and Weissing (2000)) and hence may be particularly appropriate targets for the application of the current model.

\section{References}

[1] An, M.-Y. (1998) Logconcavity versus logconvexity: A complete characterization. J. of Econ. theor. 80, 350-369.

[2] Bagnoli, M. \& Bergstrom, T. (1989) Log-concave probability and its applications. Mimeo, University of Michigan.

[3] Dawkins, R. (1989) The Selfish Gene. Oxford: Oxford University Press. 
[4] Eshel, I. \& Feldman, M (1991) The handicap principle in parent-offspring conflict: Comparison of optimality and population-genetic analyses. Am. Nat. 137, 167-185.

[5] Eshel, I., Volovik, I. \& Sansone, E. (2000) On Fisher-Zahavi's handicapped sexy son. Evol. Ecol. Res. 2, 509-523.

[6] Fudenberg, D. \& Tirole, J. (1991) Game Theory. Cambridge, Massachusetts: MIT Press.

[7] Godfray, H. C. J. (1991) Signalling of need by offspring to their parents. Nature 352, $328-330$.

[8] Godfray, H. C. J. (1995a) Evolutionary theory of parent-offspring conflict. Nature 376, $133-138$.

[9] Godfray, H. C. J. (1995b) Signaling of need between parents and young: Parent-offspring conflict and sibling rivalry. Am. Nat. 146, 1-24.

[10] Godfray, H. C. J. \& Johnstone, R. A. (2000) Begging and bleating: The evolution of parent-offspring conflict. Phil. Trans. R. Soc. Lond. B 355, 1581-1591.

[11] Grafen, A. (1990a) Biological signals as handicaps. J. theor. Biol. 144, 517-546.

[12] Grafen, A. (1990b) Sexual selection unhandicapped by the Fisher process. J. theor. Biol. 144, 473-516.

[13] Iwasa, Y. \& Pomiankowski, A. (1995) Continual change in mate preferences. Nature 377, $420-422$.

[14] Iwasa, Y., Pomiankowski, A. \& Nee, S. (1991) The evolution of costly mate preferences II: The "handicap" principle. Evolution 45, 1431-1442.

[15] Johnstone, R. A. (1995) Sexual selection, honest advertisement and the handicap principle: Reviewing the evidence. Biol. Rev. 70, 1-65. 
[16] Johnstone, R. A. (1998) Game theory and communication. In: Game Theory and Animal Behavior (Dugatkin, L. A. \& Reeve, H.-K., eds) pp. 94-117. Oxford: Oxford University Press.

[17] Johnstone, R. A. \& Grafen, A. (1992) The continuous Sir Philip Sidney game: A simple model of biological signalling. J. theor. Biol. 156, 215-234.

[18] Kilner, R. \& Johnstone, R. A. (1997) Begging the question: Are offspring solicitation behaviours signals of need? Trends Ecol. and Evol. 12, 11-15.

[19] Kirkpatrick, M. (1986) The handicap mechanism of sexual selection does not work. Am. Nat. 127, 222-240.

[20] Kirkpatrick, M. (1987) Sexual selection by female choice in polygynous animals. Ann. Rev. Ecol. Syst. 18, 43-70.

[21] Maskin, E. \& Riley, J. (1986) Existence and uniqueness of equilibrium in sealed high bid auctions. Department of Economics discussion paper 407, UCLA.

[22] Maynard Smith, J. (1976) Sexual selection and the handicap principle. J. theor. Biol. 57, 239-242.

[23] Maynard Smith, J. (1991) Honest signalling: The Philip Sidney game. Anim. Behav. 42, 1034-1035.

[24] Maynard Smith J. (1994) Must reliable signals be costly? Anim. Behav. 47, 1115-1120.

[25] Nöldeke, G. \& Samuelson, L. (1999) How costly is the honest signaling of need? J. theor. Biol. 197, 527-539.

[26] Otto, S. P. (1991) On evolution under sexual and viability selection: A two-locus diploid model. Evolution 45, 1443-1457.

[27] Pen, I. \& Weissing, F. J. (2000) Sexual selection and the sex ratio: an ESS analysis. Mimeo, University of Groningen. 
[28] Pomiankowski, A. (1987a) The costs of choice in sexual selection. J. theor. Biol. 128, 195-218.

[29] Pomiankowski, A. (1987b) Sexual selection: The handicap principle does work-sometimes. Proc. R. Soc. London B 231, 123-145.

[30] Selten, R. (1980) A note on evolutionarily stable strategies in asymmetric animal contests. J. theor. Biol. 84, 93-101.

[31] Zahavi, A. (1975) Mate selection-A selection for a handicap. J. theor. Biol. 53, 205-214.

[32] Zahavi, A. (1977) The cost of honesty (Further remarks on the handicap principle). J. theor. Biol. 67, 603-605. 


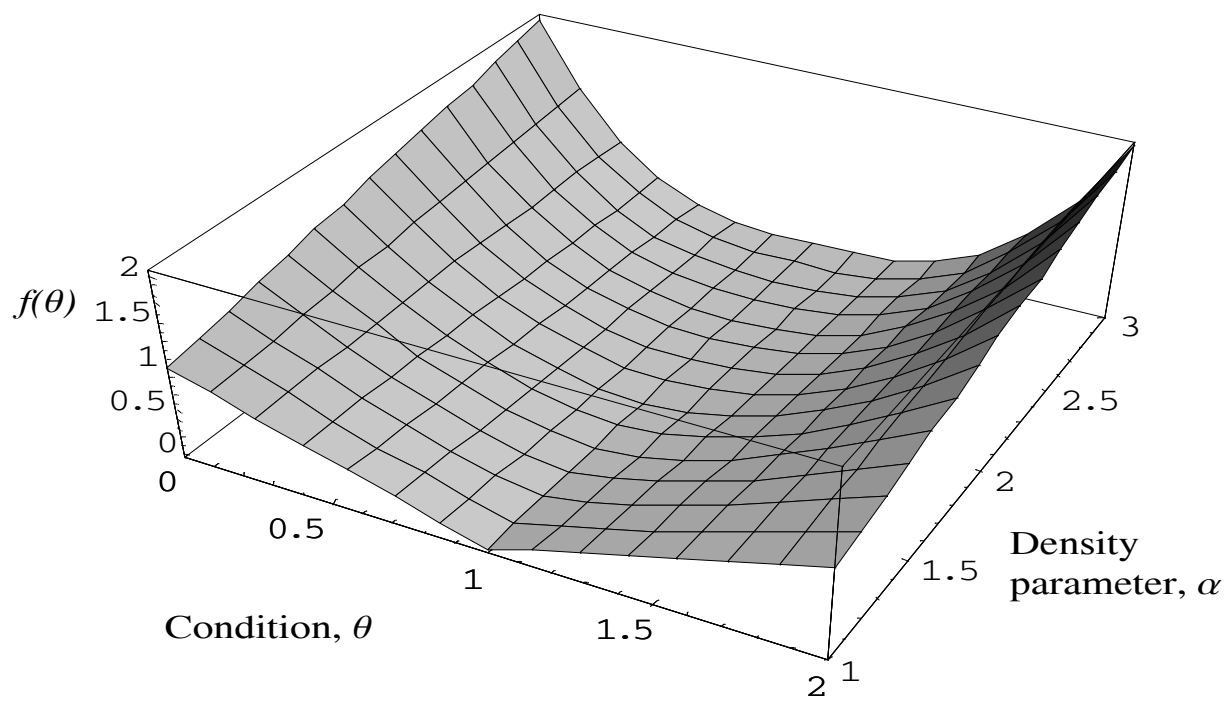

Figure 1: Density for the distribution function given in (8) and used in Fig. 2. The density is given by $f(\theta)=\frac{1}{2}(\alpha+1)(1-\theta)^{\alpha}$ when $\theta \in[0,1]$ and by $f(\theta)=\frac{1}{2}(\alpha+1)(\theta-1)^{\alpha}$ when $\theta \in[1,2]$. 

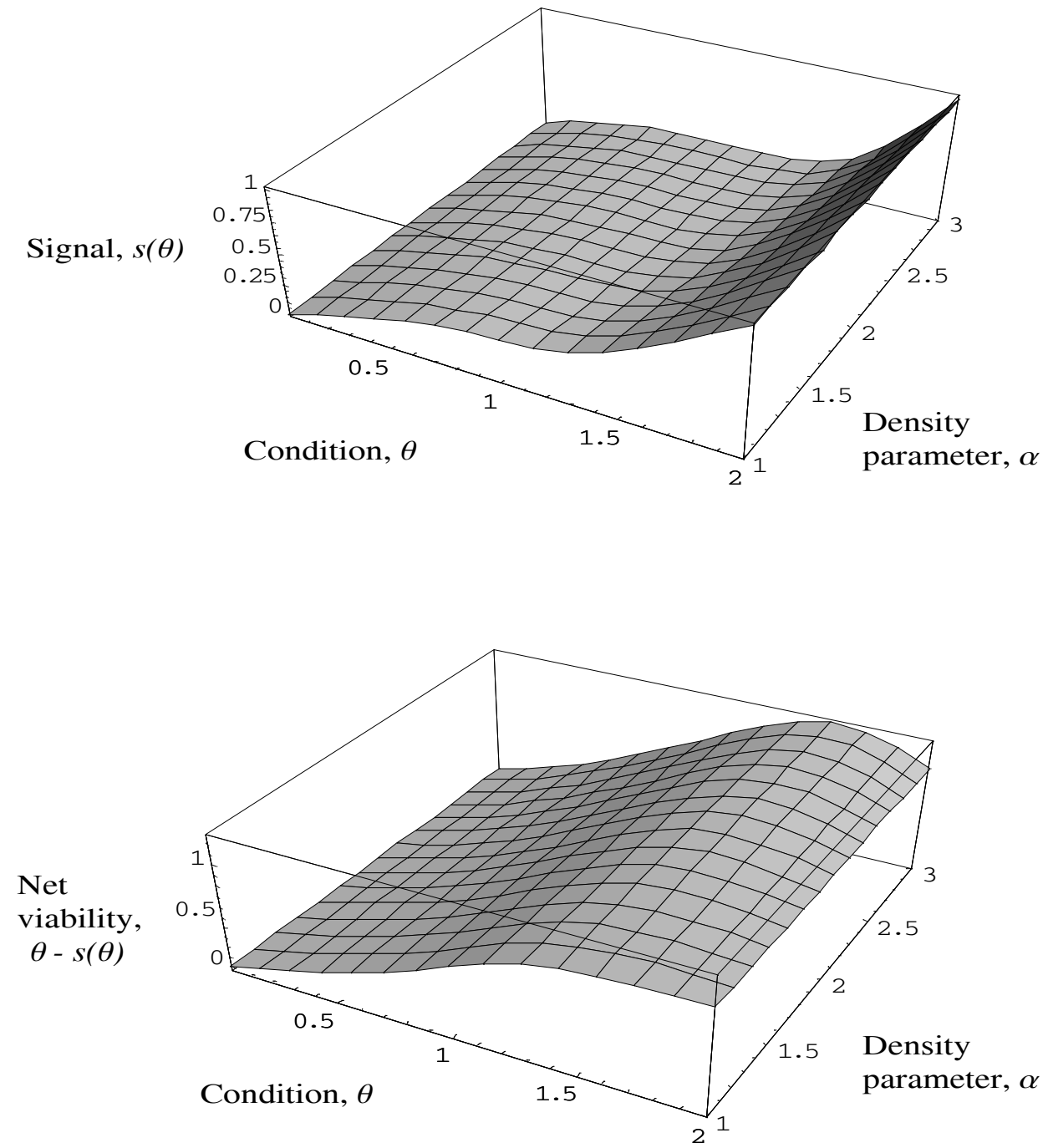

Figure 2: Illustration of a case with no signaling equilibrium. In this example, there are two males $(n=2)$ and male condition is distributed on the interval $[0, \bar{\theta}]=[0,2]$. The distribution function $F$ of male conditions is given by (8), where $\alpha \geq 0$ is a parameter. In any signaling equilibrium, the signaling function $s(\cdot)$ must be given by (5). The upper figure shows the signal $s(\theta)$ as a function of male condition $\theta$ and $\alpha$. Males in higher condition send higher signals. The lower figure shows male net viability, given by (7), as a function of $\theta$ and $\alpha$. For values of $\alpha>1$, net viability fails to be increasing in male condition, ensuring that there is no signaling equilibrium. For example, the derivative of net viability at $\theta=2$ is given by $\left(F(2)^{2}-\int_{0}^{2} F(\tilde{\theta}) d \tilde{\theta} f(2)\right) / F(2)^{2}=1-f(2)=1-\frac{1}{2}(\alpha+1)$, which is negative whenever $\alpha>1$. 